\title{
CUIDAMOS DOS OUTROS, MAS QUEM CUIDA DE NÓS? VULNERABILIDADES E IMPLICAÇÕES DA COVID-19 NA ENFERMAGEM
}

Delmair Oliveira Magalhães Luna Filha ${ }^{1}$ Beatriz de Castro Magalhães ${ }^{1}$ Mauro Mccarthy de Oliveira Silva ${ }^{1}$ Grayce Alencar Albuquerque ${ }^{1}$ https://orcid.org/ 0000-0003-1344-5669 https://orcid.org/ 0000-0002-6827-6359 https://orcid.org/ 0000-0001-8895-7760 https://orcid.otg/ 0000-0002-8726-0619

Objetivo: refletir sobre os fatores associados à vulnerabilidade do profissional de enfermagem e as repercussões na assistência durante a pandemia COVID-19. Método: ensaio teórico de cunho reflexivo, oriundo de estudos e debates referentes à vulnerabilidade dos profissionais de enfermagem, enquanto trabalhadores da saúde, e aproximação desta condição com o cenário atual de enfrentamento à pandemia da COVID-19. Resultados: a pandemia do novo coronavírus expôs a vulnerabilidade que já assolava os profissionais de enfermagem frente à desvalorização da profissão, salários baixos, subdimensionamento da equipe de enfermagem, cargas horárias exorbitantes e condições de trabalho insatisfatória, com escassez de equipamentos de proteção individual; e que em conjunto, elevam a susceptibilidade à contaminação pela COVID-19, adoecimento mental e redução da qualidade da assistência prestada. Considerações finais: com elevada vulnerabilidade ao adoecimentoecontaminação,percebe-seanecessidadeemergencialdemudançasestruturaisnoqueconcerneavalorizaçãodaenfermagem. Descritores: Equipe de Enfermagem; Pandemias; Assistência à Saúde; Saúde do trabalhador.

\section{WE CARE FOR THE OTHERS, BUT WHO CARES FOR US? VULNERABILITIES AND IMPLICATIONS OF COVID-19 IN NURSING}

Objective: to reflect on the factors associated with the vulnerability of the nursing professional and the repercussions in care during the COVID-19 pandemic. Method: a theoretical essay of reflective nature, coming from studies and discussions relating to the vulnerability of nursing professionals, while health workers, and approximation of this condition with the current scenario of coping with the COVID-19 pandemic. Results: the pandemic of the new coronavirus exposed the vulnerability that has already been ravaging nursing professionals facing the devaluation of the profession, low wages, under-dimensioning of the nursing team, exorbitant hourly loads and poor working conditions, with a scarcity of personal protective equipment, which, together, increase the susceptibility to contamination by COVID-19, mental illness and reduced quality of assistance provided. Final Considerations: with high vulnerability to illness and contamination, there is the urgent need for structural changes concerning the enhancement of nursing. Descriptors: Nursing, Team; Pandemics; Delivery of Health Care; Occupational Health.

\section{CUIDAMOS DE LOS DEMÁS, SINO ¿QUIÉN CUIDA DE NOSOTROS? VULNERABILIDADES Y CONSECUENCIAS DE LA COVID-19 EN ENFERMERÍA}

Objetivo: reflexionar sobre los factores asociados a la vulnerabilidad de los profesionales de enfermería y las repercusiones en la atención durante la pandemia COVID-19. Método: un ensayo teórico de naturaleza reflexiva, procedente de estudios y debates relativos a la vulnerabilidad de los profesionales de enfermería, en cuanto trabajadores de salud, y la aproximación de esta condición con el actual escenario de enfrentamiento de la pandemia del COVID-19. Resultados: la pandemia del nuevo coronavirus puso de manifiesto la vulnerabilidad que ha asolado a los profesionales de enfermería frente a la devaluación de la profesión, los bajos salarios, la falta de dimensionamiento del equipo de enfermería, exorbitantes cargas horarias y las malas condiciones de trabajo, con una escasez de equipamiento de protección individual, que, juntos, aumentan la susceptibilidad a la contaminación por COVID-19, enfermedad mental y disminución de la calidad de la asistencia prestada. Consideraciones finales: con un alto grado de vulnerabilidad a la enfermedad y a la contaminación, hay una necesidad urgente de cambios estructurales en lo que se refiere a la mejora de la enfermería. Descriptores: Grupo de Enfermería; Pandemias; Prestación de Atención de Salud; Salud Laboral.

${ }^{1}$ Universidade Regional do Cariri, CE.

Autor Correspondente: Grayce Alencar Albuquerque. E-mail: geycyenf.ga@gmail.com

Recebido: 23/4/2020 - Aceito: 25/5/2020 


\section{INTRODUÇÃO}

No final do ano 2019, um novo coronavírus foi nomeado como SARS-CoV-2, que produz uma doença classificada como COVID-19, sendo agente causador de uma série de casos de pneumonia na cidade de Wuhan (China), se disseminando rapidamente em todo o território mundial e em consequência disto, tendo sido declarada pela Organização Mundial da Saúde (OMS) em 30 de janeiro de 2020 como uma Emergência de Saúde Pública de Importância Internacional, o mais alto nível de alerta da Organização, e em 11 de março do mesmo ano, como uma pandemia ${ }^{(1)}$.

No mundo até o dia 20 de abril de 2020 já se somam mais de 2.314 .621 casos de infectados pela COVID-19, com um total de 157.847 mortes. No Brasil, foram confirmados até este período 36.599 casos e 2.347 mortes $^{(2)}$. Os números reforçam a alta transmissibilidade do vírus, que potencializa complicações graves, como a síndrome respiratória aguda. Estima-se que 20\% dos pacientes afetados precisarão de oxigenoterapia, muitas vezes em unidades de terapia intensiva (UTI), fundamentais para poder tratar pacientes. Isso implica no funcionamento do sistema de saúde ${ }^{(2)}$ especialmente em países subdesenvolvidos, como o Brasil.

Sabe-se que a maioria das UTI públicas no Brasil e em outros países com sistemas públicos de saúde opera em quase $100 \%$ da sua capacidade e estima-se que a média para todo o Brasil, é a existência em torno de 13 leitos de UTI por 100.000 habitantes, havendo menores quantidades no Norte e Nordeste ${ }^{(3)}$. Esta é uma situação crítica, uma vez que configura um desfecho dramático; de um lado, o aumento do número de infectados com posterior elevação do número de casos graves (mesmo diante decretos de quarentena observados) e do outro, a falta de UTI, configurando-se desta maneira, o colapso do sistema de saúde e a possibilidade de crescente número de óbitos.

Para além das superlotações em unidades de tratamento intensivo, o que exige dos governantes manejo rápido para otimização da infra-estrutura das unidades, necessita-se ter profissionais capacitados e qualificados à assistência desses pacientes, e nesse contexto, grande destaque tem tido os profissionais de enfermagem.

No Brasil, a enfermagem é reconhecida pelo Conselho Nacional de Saúde como uma das profissões da saúde, sendo regulamentada pela lei 7498/1986. Trata-se de uma profissão presente na quase totalidade das instituições de assistência à saúde e que presta um serviço essencial à vida humana ${ }^{(4)}$.

Conforme dados recentes do Conselho Federal de Enfermagem (Cofen), no primeiro trimestre de 2020, os profissionais de enfermagem totalizavam no Brasil 2.263.132, sendo a categoria profissional base para funcionamento do SUS, representando mais de $60 \%$ dos profissionais de saúde do Brasil. Neste sentido, fica claro que os profissionais de enfermagem estão na linha de frente do combate à COVID-19 e por isso, mais susceptíveis ao risco de contaminação, violências e impactos na saúde mental(5).

Sabidamente em situações de estresse, como o é em uma pandemia, com inúmeras pessoas buscando ao mesmo tempo assistência em saúde, elevam-se as chances de profissionais de enfermagem serem vitimas de violência em seus ambientes de trabalho. Além da vitimização, fatores como sensação de insegurança no ambiente de trabalho, baixa remuneração, péssimas condições de trabalho(b) e falta de Equipamentos de Proteção Individual (EPI) ${ }^{(7)}$ podem favorecer com que o profissional de enfermagem manifeste impactos negativos em sua saúde mental durante a pandemia COVID-19, com repercussões negativas na assistência.

Assim, levando-se em consideração a conjuntura atual de inserção dos profissionais de enfermagem enquanto linha de frente de combate ao novo coronavírus, este estudo objetivou refletir sobre os fatores associados à vulnerabilidades do profissional de enfermagem e as repercussões na assistência durante a pandemia.

\section{MÉTODO}

Ensaio teórico de cunho reflexivo, oriundo de estudos e debates referentes à vulnerabilidade dos profissionais de enfermagem, enquanto trabalhadores da saúde, e aproximação desta condição com o cenário atual de enfrentamento à pandemia da COVID-19, em que as reflexões propostas foram embasadas à luz (principalmente) da literatura atinente ao tema, antes e durante a pandemia do novo coronavirus.

Como não se trata de um estudo de revisão sistemática da literatura, o material utilizado foi obtido principalmente através do acesso a bancos de dados disponiveis online, sendo por meio destes acessado artigos, resoluções referentes à órgãos de conselhos de classe, normatizações técnicas frente à COVID-19 e recomendações para assistência.

A apresentação das explanações e reflexões neste artigo buscaram contemplar eixos sobre o tema, advindos de interpretações da literatura e também das impressões reflexivas dos autores. Estas interpretações foram dirigidas pela compreensão do assunto direcionadas para a sua reflexão à realidade brasileira. 
Implicações da pandemia COVID-19 no cuidado de enfermagem: vulnerabilidades ao desgaste físico, emocional e assistencial

Devido ao aumento da demanda por serviços de saúde, a pandemia do novo coronavírus escancarou, no Brasil, a realidade da superlotação do Sistema Único de Saúde (SUS), que embora esteja visível à sociedade, boa parte da população, tende a naturalizá-la, por acreditar que em um sistema público, as falhas estejam legitimidas. Ressalta-se que essa realidade se associa ao subfinanciamento crônico do SUS, que além de dificultar investimentos em estrutura, custeia de forma insatisfatória os serviços de profissionais indispensáveis à qualidade da assistência, como os profissionais de enfermagem.

Apesar de indispensáveis para a assistência à saúde, os profissionais de enfermagem ainda flutuam às margens da hegemonia biomédica, em que se percebe enfermeiros, técnicos e auxiliares de enfermagem como subalternos ao profissional médico, e não como integrantes da equipe de saúde. Assim, além do subfinanciamento do SUS, a cultura médica centrada contribui para a desvalorização da enfermagem.

A desvalorização desta categoria se traduz em vários aspectos, que vulnerabilizam o profissional, dentre eles o vínculo empregatício instável, condições de trabalho precárias, excesso de trabalho, salários minimamente incompatíveis com a carga de trabalho executada, além de escassez de EPI.

Araújo et $a^{(8)}$, apontam que as instituições de saúde têm trabalhado com um quantitativo de profissionais de enfermagem bem abaixo do preconizado, expondo dados do Nursing Activities Score (NAS), instrumento que quantifica as atividades de enfermagem em um período de 24 horas. Os dados encontrados no estudo(8) reforçam que são requeridas 20,5 horas de assistência para cada paciente/dia. No entanto, o tempo dispensando foi de 14,4 horas de atividades de enfermagem à cada paciente por dia, observando-se a necessidade de aumento no quadro laboral da enfermagem para atingir a média do NAS. Esses dados estimulam a retórica da ausência do dimensionamento adequado dos trabalhadores de enfermagem e carga horária exequivel, bandeira há muito tempo levantada pela categoria e órgãos de classe e no atual momento de enfrentamento da pandemia COVID-19, ganhando maior destaque e ameaça.

Nesse sentido, expõe-se a Medida Provisória (MP) 927, de 22 de março de 2020, editada pela presidência da república, que altera as relações de trabalho, permitindo a ampliação da jornada dos profissionais de saúde por até 24 horas e as reduções do tempo de descanso para 12 horas ${ }^{9}$. A MP reflete condutas governamentais arbitrárias não somente relacionadas aos direitos trabalhistas, como também inconsistentes à conciliação de uma assistência de qualidade, podendo culminar em efeito rebote, com aumento de contaminação de profissionais de saúde/pacientes e exaustão profissional.

Ainda, de forma a ilustrar a realidade da desvalorização da enfermagem, cabe expor a nota de repúdio que o Conselho Regional de Enfermagem do Ceará lançou no dia 20 de abril de 2020 em relação à tabela de valores de plantões a serem pagos pela Secretária de Saúde do Ceará frente a pandemia COVID-19, em que aos profissionais de enfermagem foram ofertados valores de pagamento de plantões diurnos semanais irrisórios, na qual para técnico de enfermagem e enfermeiro, respectivamente, o salário representou $6,21 \%$ e $15,48 \%$ em relação ao valor maior ofertado, que foi ao profissional médico ${ }^{(10)}$

Assim, reflete-se o quanto os profissionais de enfermagem são inferiorizados, mesmo exercendo carga horária na maioria das vezes superior a dos demais profissionais de saúde, bem como, o quanto essa categoria merece valorização, tanto pelo reconhecimento pessoal como pelo incentivo financeiro, tendo em vista o comprometimento dos profissionais de enfermagem, que mesmo enfrentando riscos à sua saúde, se destacam na luta contra o novo coronavírus.

De fato, na atual conjuntura sanitária brasileira, percebe-se uma emergência na discussão sobre as vulnerabilidades do profissional de enfermagem, posto sua atuação integral na linha de frente de combate e tratamento à COVID-19, principalmente no que se refere a atuação em hospitais. Estes são equipamentos da rede de atenção à saúde caracterizados pelo trabalho exaustivo dos profissionais de enfermagem, em função de um desequilíbrio na relação demanda-profissional e pela convivência com o sofrimento humano, culminando em esgotamento físico, intelectual e psicológico desses profissionais, que por vezes compromete a assistência à saúde ${ }^{(6)}$, tornando-os assim mais vulneráveis à violência.

Destaca-se que ao avaliar as condições de trabalho e de relacionamento a que estão submetidos os profissionais de enfermagem do Brasil com registro no Cofen, Machado et $\mathrm{al}^{(6)}$ revelam que $42,7 \%$ dos profissionais de enfermagem relataram desrespeito e maus-tratos perpetrados por usuários do serviço de saúde; $24,7 \%$ da equipe percebem seus chefes distantes e inacessiveis quando necessitam de ajuda e cerca de $30 \%$ relataram violência no trabalho, com maior preponderância da psicológica (66,5\%), seguida da institucional (17,1\%). 
Compreende-se a violência institucional de forma transversal, tanto pela possivel imposição de poder pela chefia, assim como pela falta de subsidios necessários as boas condições de trabalho, que deveriam ser ofertados pelos órgãos competentes do governo, a exemplo da falta de EPI, especialmente durante a pandemia COVID19, o que implica em redução da segurança profissional diante da assistência, especialmente daqueles lotados em UTI, setor em que há contato com pacientes gravemente comprometidos pela COVID-19, por necessitarem de suporte ventilatório.

Alguns autores ${ }^{(11)}$ expõem que nesse setor os riscos preponderantes são i) psicológicos e emocionais, relacionados ao estresse do ambiente de trabalho; ii) ergonômicos, principalmente no que se refere ao manuseio do paciente no leito e iii) biológicos, relacionados ao contato com secreções, aerossóis, perfurocortantes, expondo-se o profissional a microorganismos patogênicos.

Assim, no que diz respeito ao panorama de contaminação da classe de enfermagem, compreende-se a reflexão acerca dos riscos biológicos principalmente diante da escassez de EPI. Destaca-se que desde a caracterização da pandemia se intensificaram as medidas de sensibilização quanto ao uso destes insumos, com atenção especial às máscaras cirúrgicas que devem ser utilizadas preferencialmente em todo ambiente hospitalar, havendo ou não suspeita de pacientes com COVID-19; e às mascaras especiais N95 em ambientes de UTI e em todo procedimento gerador de aerossóis ${ }^{(1)}$.

Entretanto, além da sensibilização, é necessário que a enfermagem tenha acesso a estes insumos. A falta de EPI condiciona os profissionais a reutilizarem máscaras N95 e utilizarem máscaras de material duvidoso. O Cofen até a data de 17/04/2020 já sinalizava 4,8 mil denúncias por falta desses equipamentos ${ }^{(5)}$. Destaca-se que o Ministério da Saúde tentou pleitear a compra desses materiais com a China, que concentra a maior produção de equipamentos para a saúde, no entanto, a venda para o Brasil foi embargada em detrimento de uma demanda para os Estados Unidos ${ }^{(7)}$.

Fato é, que se pode visualizar um cenário de agravo às condições de trabalho dos profissionais de enfermagem, pois além das vulnerabilidades já firmadas, somam-se alterações tanto na rotina profissional como pessoal, em que se destacam questões de cunho familiar, como a preocupação na transmissibilidade do vírus para entes queridos após retorno aos domicílios findados os plantões. Revela-se ainda, que os hospitais não ofertam infraestrutura digna para descanso no local de trabalho, o que pode contribuir ainda mais para a exaustão profissional(6).
Esta situação implica em desgastes físicos e emocionais, pois após uma longa e exaustiva jornada de trabatho, o profissional acaba tendo que se abster do conforto do lar e do carinho dos familiares, mantendo-se em isolamento, como é elucidado em reportagem da BBC News (10 de abril de 2020) em que um técnico de enfermagem revela "dormir no terraço para não infectar a mãe"12. Essa realidade incita uma reflexão sobre as relações familiares fragilizadas em tempos de pandemia e que podem manter relação de causa e efeito com o estresse profissional. Ainda, o estresse corrobora para vulnerabilizar as relações interpessoais, o que causa certa preocupação, pois nesse momento, de substancial aumento do estresse laboral, a carência de relações interpessoais e apoio familiar e social corroboram para o adoecimento mental, que de forma somatizada, repercute também em adoecimento físico.

As demandas decorrentes do adoecimento mental e físico da enfermagem exigem suporte dos serviços de saúde para seu devido enfrentamento. No entanto, conforme afirma Machado et al( $^{(6)}$ cerca de $49,6 \%$ dos profissionais de enfermagem não recebem assistência à saúde quando precisam. No cenário atual, isso é bastante preocupante, mediante a taxa de contaminação nos profissionais de enfermagem e as mortes por causa da COVID-19. Segundo matéria do Campo Grande News, conforme Cofen (20/04/2020), através do Observatório de Enfermagem, em todo Brasil, contabiliza-se 34 mortes na categoria de enfermagem por COVID-19 entre confirmados e suspeitos e 5.174 profissionais com sintomas ou diagnóstico da doença afastados ${ }^{(13)}$.

Diante das discussões realizadas sobre as vulnerabilidades dos profissionais de enfermagem e somando-se estas ao excesso de trabalho, descanso comprometido e pressão psicológica profissional e pessoal, infere-se que as elevadas chances de contaminação pelo novo coronavírus possam acontecer por desatenção às medidas preventivas dentro e fora da instituição de saúde, tendo em vista o esgotamento profissional.

Se a curva de contaminação aumenta e os profissionais não tem EPI ou condições necessárias à proteção, a tendência é que mais profissionais sejam contaminados e afastados; o que gera um ciclo vicioso entre contaminação dos profissionais, possivel transmissão a outros profissionais e pessoas em geral, bem como, afastamento de profissionais qualificados, convergindo-se para o que têm-se denominado como colapso do SUS ${ }^{(5)}$.

Esse colapso pode ocorrer também aliado ao baixo número de leitos de UTI e baixo quantitativo de profissionais 
de enfermagem por demanda. A Agencia Nacional de Vigilância em Saúde ${ }^{14}$ recomenda que UTI possuam um técnico de enfermagem para cada dois pacientes e um enfermeiro para cada oito leitos. Essa recomendação reforça a crítica acerca do subfinaciamento crônico do SUS, devido ao baixo investimento em recursos humanos, bem como, a desvalorização dos profissionais de enfermagem, tendo em vista que não é de hoje que estes profissionais trabalham 40 horas semanais ou mais e cobrem um número de pacientes bem maior que o recomendado.

A crise atual na saúde brasileira, possui cunho histórico, o SUS foi instituido, mas nunca consolidado, passando por ataques e tentativas de desmonte, em que vale destacar o congelamento de orçamento público para a saúde durante 20 anos, definida pela Emenda Constitucional 95 de 2016; além de indisponibilidade de condições objetivas para a sustentabilidade econômica e científico-tecnológica do sistema ${ }^{15}$. Assim, tanto a escassez de investimentos em recursos humanos, como os da enfermagem (no sentido de reconhecer a indissociabilidade dessa profissão na qualidade da assistência), quanto a escassez de financiamento de materiais e estrutura, corroboram para a situação atual em que se encontra o Sistema Único de Saúde e esta categoria profissional frente a pandemia.

Nessa perspectiva, a crise na saúde desvelou uma carência que há muito tempo deveria estar sanada, como destaca o Cofen (17/04/2020) sobre a necessidade de contratação emergencial de profissionais de enfermagem e remanejamento da força de trabalho, com especial atenção aos profissionais que se enquadram nos grupos de risco à contaminação COVID-19, como idosos, diabéticos e hipertensos, que devem ser afastados ${ }^{(5)}$

Ressaltam-se que as hospitalizações continuam ocorrendo não somente pela infecção do novo coronavírus, mas também por outras condições de saúde. Assim, o déficit de profissionais de enfermagem já existente (com destaque aos contaminados pela COVID-19), aliado aos atuais afastamentos e às condições que comprometem a qualidade do trabalho na enfermagem, trazem implicações para a qualidade da assistência ao usuário, gerando maiores gastos ao SUS, como por exemplo, ocorrência de eventos adversos e normas de segurança do paciente comprometidas.

A contratação emergencial a que o Cofen se refere é de fundamental relevância, posto que não raro, profissionais que estão expostos à péssimas condições de trabalho tendem a comprometer sua assistência mediante desgaste físico e emocional. É possível que essa contratação não precisasse ocorrer emergencialmente se as necessidades da enfermagem tivessem sido priorizadas há mais tempo, pois percebe-se que hoje tem-se um quadro de profissionais já esgotados, precisando lidar com o enfrentamento de uma crise na saúde, ao mesmo tempo que lidam com problemas que já os assolavam antes da pandemia, como é o caso da busca pela valorização profissional e dignidade no exercício da profissão.

\section{Limitações do estudo}

A escassez de estudos publicados sobre os impactos da COVID-19 no que se refere à Enfermagem é considerada uma limitação, tendo em vista a necessidade de discussão pautada em artigos.

\section{Contribuições para a prática}

Esse estudo contribui para elucidação de questões relevantes sobre as condições de trabalho atual da Enfermagem e para colaboração no suprimento de estudos sobre o novo coronavírus, considerando de forma ampliada o seu impacto. Dessa forma, a reflexão pode incitar o pensamento crítico sobre a readequação do exercício da enfermagem no sentido de condicionar qualidade ao trabalho, repercutindo positivamente na saúde do usuário e do trabalhador.

\section{CONSIDERAÇÕES FINAIS}

A pandemia COVID-19 tornou ainda mais visivel a desvalorização da Enfermagem, que ocorre não somente pelo subfinanciamento do SUS, mas principalmente pela cultura essencialmente biomédica, percebida através da discrepância de salários e carga horárias entre profissionais destas categorias. Além disso, a Enfermagem ainda enfrenta a exposição aos riscos ocupacionais, com destaque aos biológicos, especialmente diante escassez de EPI.

Percebe-se as péssimas condições de trabalho da Enfermagem como um problema de saúde pública, que impacta diretamente na segurança do paciente. Assim, compreende-se a necessidade emergencial de uma mudança, não somente em relação à contratações para substituição de profissionais afastados, mas também uma mudança estrutural no sentido de promover a valorização profissional pelo reconhecimento pessoal, financeiro e pela oferta de melhores condições de trabalho. Somente assim é possível vislumbrar um cenário em que os profissionais de enfermagem estejam protegidos $e$ aptos a garantirem a qualidade da assistência, dispondo de tempo suficiente à cada paciente, ao mesmo tempo que preservam sua saúde física e emocional nestes tempos de pandemia. 


\section{CONTRIBUIÇÕES DOS AUTORES}

Delmair Oliveira Magalhães Luna Filha: Realizou contribuições substanciais na concepção do estudo, coleta, análise e interpretação dos dados; Beatriz de castro Magalhães: Realizou contribuições substanciais na coleta, análise e interpretação dos dados, bem como na redação e revisão crítica do manuscrito; Mauro Mccarthy de Oliveira Silva: Realizou contribuições substanciais na redação e revisão crítica do manuscrito; Grayce Alencar Albuquerque: Realizou contribuições substanciais na concepção do estudo, redação e revisão crítica do manuscrito, bem com, na aprovação da versão final a ser publicada.

\section{REFERÊNCIAS}

1. Organização Panamericana da Saúde (OPAS) Brasil. Folha informativa: COVID-19 (Doença causada pelo coronavirus). Brasilia (DF); 17 de abril 2020. Available from: https://www. paho.org/bra/index.php?option=com_content\&view=article\&i$\mathrm{d}=6101$ :COVID19\&Itemid $=875$

2. Organização Mundial de Saúde (OMS). Coronavirus disease 2019 (COVID-19) Situation Report - 91. Genebra. 2020. Available from: https://www.who.int/docs/default-source/coronaviruse/situation-reports/20200420-sitrep-91-COVID-19. pdf?sfvrsn=fcfO670b_4

3. Bravo JAM. Análise da capacidade instalada de terapia intensiva no municipio do rio de janeiro frente à infecção respiratoria aguda grave (SARI): preparação para o enfrentamento de pandemias. Dissertação. Fundação Oswaldo Cruz Instituto Nacional de Infectologia Evandro Chagas. Rio de janeiro, 2016. 110p. Disponivel em: https://www.arca.fiocruz.br/bitstream/ icict/25390/2/jorge_bravo_ini_mest_2016.pdf

4. Conselho Federal de Enfermagem. Lei 7.498 de 25 de junho d 1986. Dispõe sobre a regulamentação do exercício da enfermagem e dá outras providências. 1986. Disponivel em: http://www.cofen.gov.br/lei-n-749886-de-25-de-junhode-1986_4161.html

5. Conselho Federal de Enfermagem. Brasil tem 30 mortes na Enfermagem por COVID-19 e 4 mil profissionais afastados. 17 de abril de 2020. Disponivel em: http://www.cofen.gov.br/brasil-tem-30-mortes-na-enfermagem-por-COVID-19-e-4-mil-profissionais-afastados_79198.html

6. Machado MH, Santos MR dos, Oliveira E, Wermelinger M, Vieira M, Lemos W et al. Condições de trabalho da enfermagem. Enferm. Foco 2016; 7 (esp): 63-76.

7. Conselho Federal de Enfermagem. Cancelamento de compra de EPI preocupa Cofen. 02 de abril 2020. Disponivel em: http:// www.cofen.gov.br/cancelamento-de-compra-de-epis-preocupa-o-cofen_78598.html.

8. Araújo TR, Menegueti MG, Auxiliadora-Martins M, Castilho V, Chaves LDP, Laus AM.Financial impact of nursing professionals staff required in an Intensive Care Unit. Rev. Latino-Am. Enfer- magem. 2016:24:e2818. DOI: http://dx.doi.org/ 10.1590/15188345.1274 .2818

9. Brasil. Presidência da República. Medida provisória № 927, de 22 de março de 2020. Dispõe sobre as medidas trabalhistas para enfrentamento do estado de calamidade pública e da emergência de saúde pública de importância internacional decorrente do coronavirus (COVID-19), e dá outras providências. Disponivel em: http://www.planalto.gov.br/ccivil_03/_ato20192022/2020/Mpv/mpv927.htm

10 Conselho Regional de Enfermagem do Ceará. Nota de repúdio contra os valores ofertados pelo Governo do Ceará aos técnicos de Enfermagem e enfermeiros que atuam no combate à COVID-19. 20 de abril de 2020. Disponivel em: http://www. coren-ce.org.br/nota-de-repudio-6/

11 Nazario EG, Camponogara S, Dias GL. Riscos ocupacionais e adesão a precauções-padrão no trabalho de enfermagem em terapia intensiva: percepções de trabalhadores. Revista Brasileira de Saúde Ocupacional. 2017 [acessado em 17 abril 2020]; 42(e7); 1-11. Doi: https://doi.org/10.1590/2317-6369000009216

12 BBC News Brasil. Durmo no terraço para não infectar minha mãe. 10 de abril de 2020. Disponivel em: https://www.bbc.com/ portuguese/brasil-52234733

13 Campo Grande News. Conselho Federal de Enfermagem contabiliza mais de 5 mil afastamentos de profissionais por conta do novo coronavírus. 20 de abril de 2020 às 17:08. Disponivel em: https://www.campograndenews.com.br/brasil/cidades/em-ms-COVID-19-afastou-12-profissionasi-da-enfermagem.

14 Brasil. Ministério da Saúde (BR). Agencia Nacional de vigilância em Saúde. Resolução - RDC n7, de 24 de fevereiro de 2010. Dispõe sobre os requisitos básicos para funcionamento de unidades de terapias intensivas e dá outras providências. Disponivel em: https://www.coffito.gov.br/nsite/?p=3377

15 Paim, JS. Sistema Único de Saúde (SUS) aos 30 anos. Ciênc. saúde coletiva [Internet]. 2018 June [cited $2020 \mathrm{Apr}$ 18]; 23(6): 1723-1728.Doi: https://doi. org/10.1590/1413-81232018236.09172018. 